\title{
One Usage of Geogebra in Enhancing Pre-service Mathematics Teachers' Content Knowledge
}

\author{
Karmelita Pjanić ${ }^{1}$ and Edin Liđan ${ }^{2}$
}

\begin{abstract}
A wide range of mathematical ideas could be used to develop and justify a formula for calculating the area of trapezoid. Those ideas lead to different strategies for finding out area of trapezoid that we classify in three groups: decomposing, enclosing and transforming strategies. Those strategies should be part of mathematics content knowledge for teaching. In this study we trace a change in structure of mathematics content knowledge of nine pre-service mathematics teachers as a result of using GeoGebra applets that visualize different approaches in finding out the area of trapezoid. We argue that engaging pre-service mathematics teachers to develop and justify formula for calculating the area of trapezoid using GeoGebra applets is a worth task that enhances pre-service mathematics teachers' content knowledge for teaching. Our experiment confirmed that the use of Geogebra encourage pre-service mathematics teachers to uncover new ideas that lead them towards clearer justifications and easier way of proving formula for area of trapezoid.
\end{abstract}

Keywords: Area of trapezoid, GeoGebra, content knowledge for teaching

DOI: $10.16949 /$ turcomat. 78085

\section{Introduction}

Solid teachers' mathematics content knowledge and their creativity, as well as the freedom to develop new ideas with pupils are equally required to make teaching of mathematics succesful. Teachers' mathematical knowledge for teaching is most profoundly manifested in numerous tasks of teaching. These tasks include analysing pupils' mathematical work and thinking, selecting, designing and modifying mathematical tasks and problems, establishing and revising mathematical goals for pupils, assessing and using resources and tools for teaching, explaining mathematical ideas and solving mathematical problems, as well as building and supporting mathematical community and discourse (Somayajulu, 2012) .

Aforementioned tasks of teaching and inovative mode of working with pupils need to be applied with all mathematics topics. Geometry and measurement offer teachers diverse opportunities to select, design and modify mathematical tasks and problems, assess and use resources and tools for teaching. In particular, in process of teaching contents of area of geometrical figures, teacher is posing problems, asking questions that build on pupils' thinking, and is encouraging students to explore different solutions. Fourthermore, pupils are asked to justify their answers and solutions and to prove conjectures. Those teachers who are aware of complexity of mathematical concepts that they teach as well as of principles of learning know that in teaching about area, it is not sufficient to give the pupils the pertinent formulas and have them merely compute the areas of various polygons.

\footnotetext{
${ }^{1}$ Prof. Dr., University of Bihać, Faculty of Education, kpjanic@gmail.com

${ }^{2}$ MA., University of Bihać, Faculty of Education, lidjan_edin@ hotmail.com
} 
Instead, they put emphasis on lesson design where pupils should develop an understanding of the concept of area so they can reason about the relationships between shapes to determine area. In order to implement efective lesson design using apropriate resources and tools teachers need to, based on their content knowledge, percieve as many different approaches and possible justifications in acquairing the concept of area.

During the primary school, pupils explore and develop formulas for the areas of square and rectangle, and at the same time are introduced to measures for area. Usually, in higher grades of elementary school (i.e. during middle school), the final forms of formulas for the area of triangle, parallelogram and trapezoid are just presented to pupils. Although pupils often do not get a chance to discover the formula for the area of a trapezoid in their mathematics classroom, when given an opportunity pupils can come up with multiple ways of justifying or discovering this formula (Manizade, 2012; Wanko, 2005; Young, 2010). So, it is a worthwhile task to ask pupils to figure out how to find the area of a trapezoid and to justify their method. However, teachers' confidence with different approaches which reveal this formula is prerequisite for asking the pupils to do the same task. Teachers' mathematical content knowledge for teaching should be strong enough to let them iniciate pupils' explorations of multiple ways in discovering and justifying formula for area of trapezoid. On the other hand, the use of computer dinamic software may support this iniciative.

As there is a wide range of mathematical ideas that could be used to develop and justify a formula for calculating the area of a trapezoid, in this study we trace the change in structure of mathematics content knowledge of pre-service mathematics teachers as a result of using GeoGebra applets that visualize different approaches in finding out the area of a trapezoid.

\section{Strategies of developing the formula for the area of trapezoid}

The definition of a trapezoid given in mathematics textbooks in Bosnia and Herzegovina is as follows: A trapezoid is a quadrilateral with exactly one pair of parallel sides. Pupils are learning how to calculate the area of trapezoid in eight grade of elementary school. The most common approach in teaching the area of trapezoid is to present the formula to pupils without any justification. However, development and justification of the formula for the area of trapezoid can be builded using a wide range of mathematics concepts and ideas. Different combinations of ideas and concepts bring into strategies for the development and justification of formula of the area of a trapezoid. Those strategies could be separated into three types (Manizade \& Mason, 2012):

Type 1: decomposition.

Type 2: enclosure.

Type 3: transformation. 
Type 1 strategy of building up the formula for the area of a trapezoid includes decomposition of a trapezoid into triangles or combination of the triangles, rectangles, and parallelograms. Then the area of a trapezoid can be found as a sum of the areas of aforementioned shapes. Figures $1-4$ show different decompositions of trapezoid.

1a) Diagonal $D B$ decomposes the trapezoid $A B C D$ into triangles $A B D$ and $B C D$. The area of $A B C D$ is equal to the sum of areas of triangles $A B D$ and $B C D$.

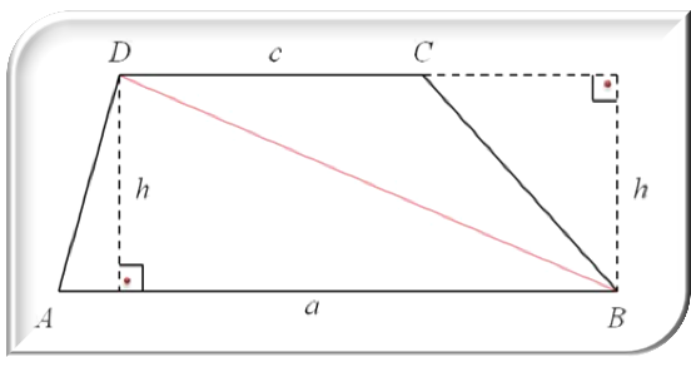

$$
\begin{aligned}
P_{A B C D} & =P_{A B D}+P_{B C D} \\
& =\frac{a h}{2}+\frac{c h}{2} \\
& =\frac{(a+c) h}{2}
\end{aligned}
$$

Figure 1. Decomposition of a trapezoid into two triangles

1b) Segment $D E$ decomposes the trapezoid $A B C D$ into the triangle $A E D$ and the parallelogram $E B C D$. The area of $A B C D$ is equal to the sum of areas of the triangle $A E D$ and the parallelogram $E B C D$.

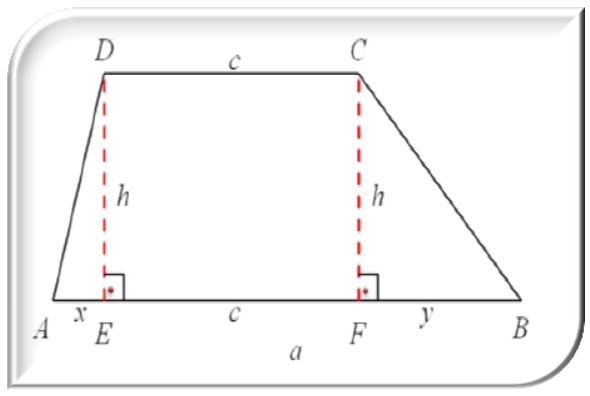

$$
\begin{gathered}
P_{A B C D}=P_{\triangle A E D}+P_{E B C D} \\
=\frac{(a-c) h}{2}+c h \\
=\frac{a h-c h+2 c h}{2} \\
=\frac{(a+c) h}{-}
\end{gathered}
$$

Figure 2. Decomposition of a trapezoid into triangle and parallelogram

1c) Altitudes $D E$ and $C F$ decompose the trapezoid $A B C D$ into the rectangle $E F C D$ and the right triangles $A E D$ and $F B C$. The area of $A B C D$ is equal to the sum of areas of the rectangle $E B C D$ and triangles $A E D$ and $F B C$. 


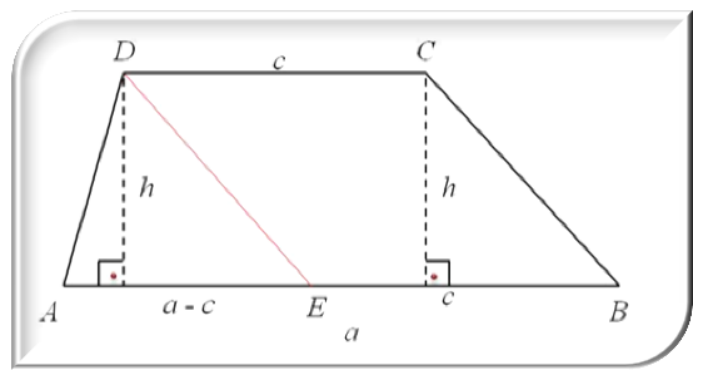

$$
\begin{aligned}
P_{A B C D}= & P_{D E F C}+\left(P_{\triangle A E D}+P_{\triangle F B C}\right) \\
& =c h+\frac{x h}{2}+\frac{y h}{2} \\
& =c h+\frac{(x+y) h}{2} \\
& =c h+\frac{(a-c) h}{2} \\
& =\frac{(a+c) h}{2}
\end{aligned}
$$

Figure 3. Decomposition of a trapezoid into two right triangles and rectangle

1d) Let $E$ is the midpoint of the base $A B$ of the trapezoid $A B C D$. Segments $D E$ and $C E$ decompose the trapezoid $A B C D$ into triangles $A E D, E B C$ and $D E C$. The area of $A B C D$ is equal to the sum of areas of triangles $A E D, E B C$ and $D E C$.

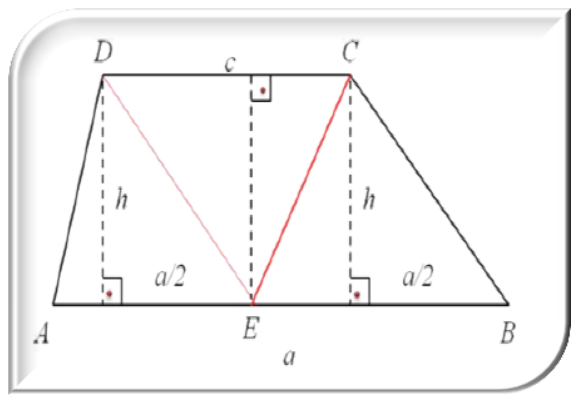

$$
\begin{aligned}
P_{A B C D} & =P_{A E D}+P_{E C D}+P_{E B C} \\
& =\frac{\frac{a}{2} h}{2}+\frac{c h}{2}+\frac{\frac{a}{2} h}{2} \\
& =\frac{(a+c) h}{2}
\end{aligned}
$$

Figure 4. Decomposition of a trapezoid into three triangles

The second type of strategy for giving form of formula for the area of a trapezoid includes enclosure of the original trapezoid into a constructed larger shape, such as a rectangle, or parallelogram, or a triangle. Then the area of a trapezoid can be found by subtracting the area of additional parts from a constructed larger shape. Figures $5-7$ illustrate strategy of enclosure.

$2 \mathrm{a})$ Using the properties of a parallelogram we can enclose the trapezoid $A B C D$ into the parallelogram $A B E D$. The area of $A B C D$ is equal to the difference of areas of the parallelogram $A B E D$ and the triangle $B E C$. 


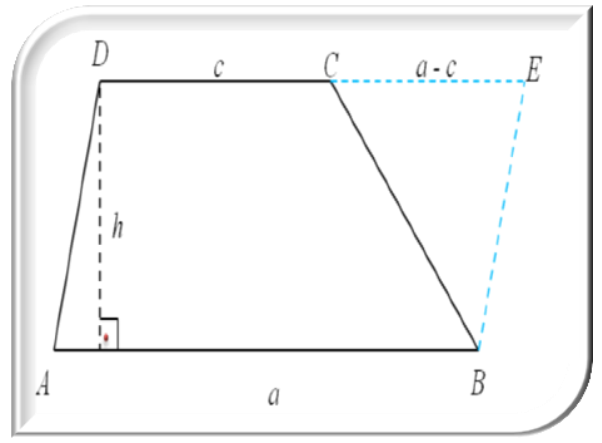

$B E \| A D$

$D E \| A B$

$$
\begin{aligned}
P_{A B C D} & =P_{A B E D}-P_{\triangle B E C} \\
& =a h-\frac{(a-c) h}{2} \\
& =\frac{(a+c) h}{2}
\end{aligned}
$$

Figure 5. Enclosure of a trapezoid into paralelogram

2b) Let $A E=A B+B E$, where $B E=D C$ and $D F=D C+C F$, where $C F=A B$. Using the properties of a transversal to parallel lines it is easy to prove that trapezoids $A B C D$ and $C F E B$ are congruent and form the parallelogram $A E F D$.

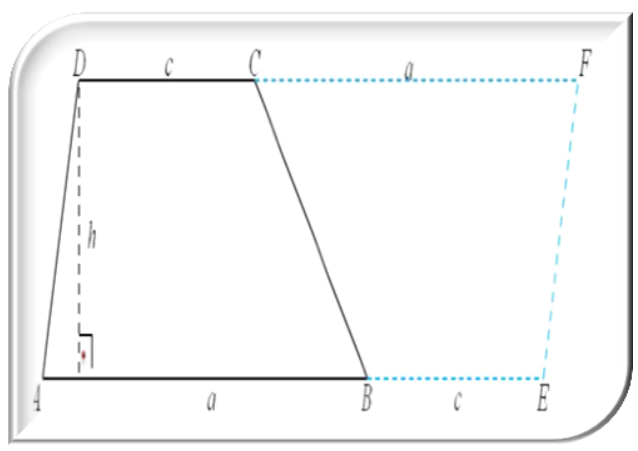

$$
\begin{gathered}
A E=a+c \\
B F=c+a \\
A E \| D F \\
E F \| A D \\
P_{A B C D}=\frac{1}{2} P_{A E F D}=\frac{(a+c) h}{2}
\end{gathered}
$$

Figure 6. Enclosure of a trapezoid into paralelogram

2c) By extending the legs $A D$ and $B C$ of the trapezoid $A B C D$ we can enclose the trapezoid into the triangle $A B E$. The area of $A B C D$ is equal to difference of areas of similar triangles $A B E$ and $D C E$. 


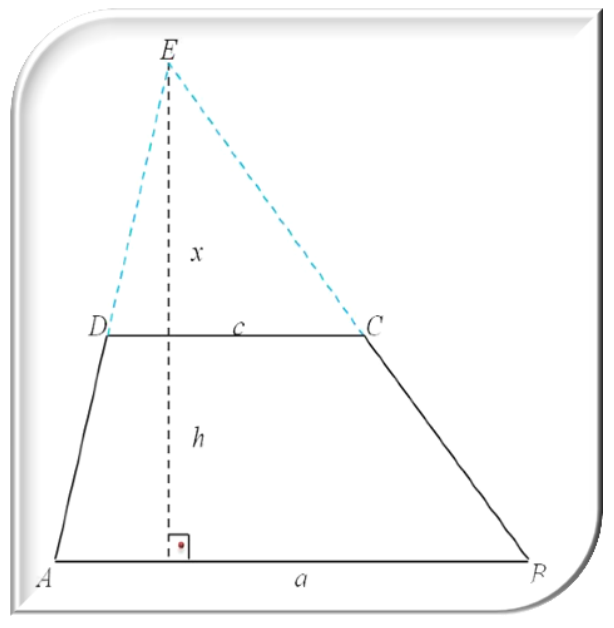

$$
\begin{gathered}
p_{A D} \cap p_{B C}=\{E\} \\
\text { Thales theorem: } \\
x: c=(x+h): a \\
\Rightarrow a x=c(x+h) \\
P_{A B C D}=P_{\triangle A B E}-P_{\triangle D C E} \\
=\frac{a(h+x)}{2}-\frac{c x}{2} \\
=\frac{a h+a x-c x}{2} \\
=\frac{a h+c x+c h-c x}{2} \\
=\frac{(a+c) h}{2}
\end{gathered}
$$

Figure 7. Enclosure of a trapezoid into triangle

2d) The trapezoid $A B C D$ is enclosed into the rectangle $A B F E$ with dimensions $a$ and $h$. The area of $A B C D$ is equal to the difference of the area of rectangle $A B F E$ and sum of areas of right triangles $A D E$ and $B F C$.

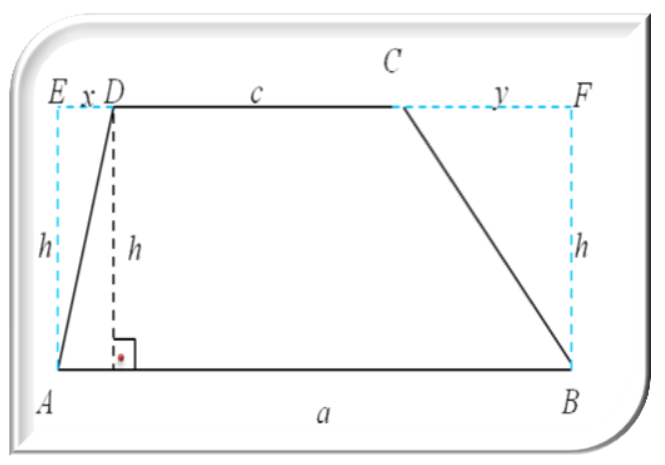

$$
\begin{aligned}
& A E=B F=h \\
& \begin{aligned}
a=E F & =E D+D C+C F \\
& =D C+(E D+C F) \\
& =c+(x+y) \\
\Rightarrow x+y & =a-c \\
P_{A B C D} & =P_{A B F E}-P_{\triangle A D E}-P_{\triangle B F E} \\
& =P_{A B F E}-\left(P_{\triangle A D E}+P_{\triangle B F E}\right) \\
& =a h-\frac{(a-c) h}{2} \\
& =\frac{(a+c) h}{2}
\end{aligned}
\end{aligned}
$$

Figure 8. Enclosure of a trapezoid into rectangle

The third type of strategy derives the formula for the area of a trapezoid by using transformational geometry. Here, ideas of rotation and symetry supported with concept of congruence are used. Figures $8-10$ present transformation strategy. 
3a) Let $O$ is midpoint of leg $B C$ of the trapezoid $A B C D$. Central symmetry of the trapezoid $A B C D$ in respect to $O$ gives the trapezoid $A^{\prime} C B D^{\prime}$. In this manner we obtain the parallelogram $A D^{\prime} A^{\prime} D$ of base $a+c$ and altitude $h$.

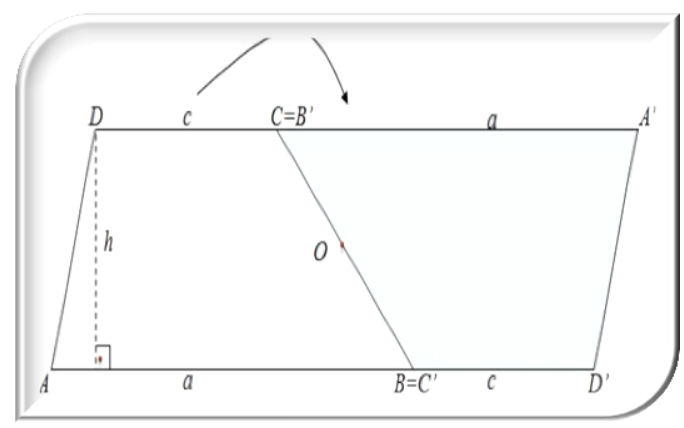

$$
\begin{aligned}
& \operatorname{Rot}(0,-180) A B C D \rightarrow A^{\prime} C B D^{\prime} \\
& \Rightarrow P_{A D^{\prime} A^{\prime} D}=2 P_{A B C D} \\
&=\frac{(a+c) h}{2}
\end{aligned}
$$

Figure 9. The parallelogram obtained by rotation of a trapezoid

$3 b)$ Let $\mathrm{EF}$ is the midline of the trapezoid $A B C D$. Thales theorem implies that $\mathrm{DG}=\mathrm{GH}=\frac{1}{2} D H$. Central symmetry of the trapezoid $E F C D$ in respect of $\mathrm{F}$ gives the trapezoid $E^{\prime} F C D^{\prime}$. In this manner the trapezoid $A B C D$ is transformed into the parallelogram $A D^{\prime} E^{\prime} E$.

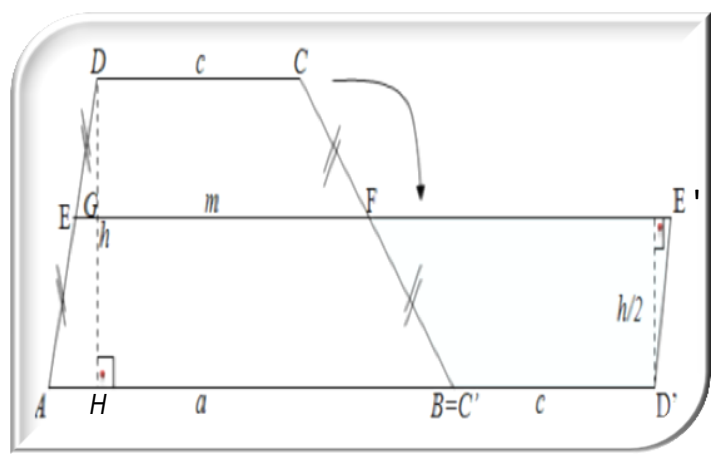

$$
\begin{gathered}
\text { Rot }\left(F,-180^{\circ}\right) E F C D \rightarrow E^{\prime} F B D^{\prime} \\
\text { Tales theorem: } \\
D G=G H=\frac{1}{2} D H \\
P_{A B C D}=P_{A B F E}+P_{E F C D} \\
=P_{A B F E}+P_{B D^{\prime} E^{\prime} F} \\
=P_{A D^{\prime} E^{\prime} E}=\frac{(a+c) h}{2}
\end{gathered}
$$

Figure 10. Transformation of a trapezoid into parallelogram

3c) Let $E$ is the midpoint of leg $B C$ of the trapezoid $A B C D$. Central symmetry of the triangle $D C E$ in respect to $E$ gives the triangle $D^{\prime} B E$. In this manner the trapezoid $A B C D$ is transformed into the triangle $A D^{\prime} D$. 


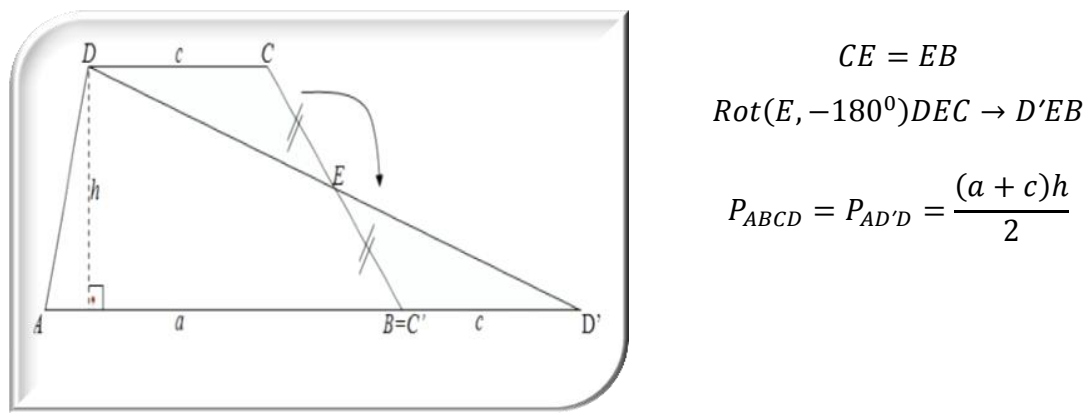

Figure 11. Transformation of a trapezoid into triangle

3d) Let $E$ and $F$ are midpoints of legs $A D$ and $B C$, respectively. Central symmetry of the triangle $A G E$ in respect to $E$ gives the triangle $D G^{\prime} E$. Central symmetry of the triangle $H B F$ in respect to $F$ gives the triangle $H^{\prime} C F$. In this manner the trapezoid $A B C D$ is transformed into the rectangle $G H H^{\prime} G$ '.

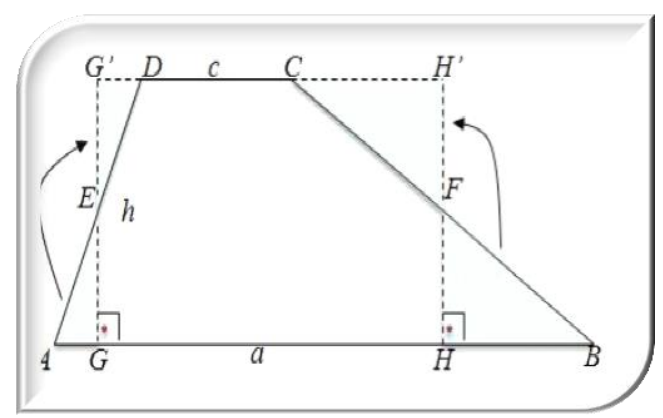

$$
\begin{gathered}
\operatorname{Rot}\left(E,-180^{\circ}\right) A G E \rightarrow D G^{\prime} E \\
\operatorname{Rot}\left(F, 180^{\circ}\right) H B F \rightarrow H^{\prime} C F \\
E F=m=\frac{a+c}{2} \\
P_{A B C D}=P_{G H H^{\prime} G^{\prime}}=\frac{(a+c) h}{2}
\end{gathered}
$$

Figure 12. Transformation of a trapezoid into rectangle

Beside that those three types of strategies bring into formula for the area of a trapezoid, they also offer justification of the formula.

\section{Visualisation and justification of the formula for the area of a trapezoid - usage of Geogebra}

In geometry studies it is not enough just to recognize geometric objects in a certain position, but to perceive their properties based on definitions as well as to be able to recognize, apply and combine geometric objects in different kinds of tasks and problems. Well-developed sense and skills of spatial visualization and orientation are very important in geometry studies. Visualization of geometric objects represents a useful way to adopt 
abstract geometric concepts, and an important factor in demonstrating the features and connections between them. Visualization is not just math appreciation through pictures (Zimmerman \& Cunningham, 1991). However, it deepens the understanding, serves as a reliable path to solving the problems and enables creative and innovative ideas that lead to solution. Hitt Espinosa (1997) suggests that the visualization of mathematical concepts is not trivial cognitive activity: to visualize does not mean the same thing as to see. In his opinion visualization represents the ability to create rich, mental images with which one can manipulate in his mind, creating different representations of mathematical concepts, and if necessary, use paper or computer, represents visualization.

Beside the visualization, justification, proof and proving have crutial role in school geometry. The teacher's role is to encourage pupils' understanding of the purpose of proof in mathematics. Studies have shown that mathematics teachers using visual tools can achieve a higher level of understanding of the mathematical (geometry) concepts and therefore upgrade their content knowledge for teaching. Also, usage of visual tools encourages teachers to uncover new ideas that would lead them towards clearer justifications and easier way of proving.

On the trail of this is the GeoGebra applet that is offered to students during our research. The aim of applet is not only enable students to see figures but to "see“ and reveal ideas behind the figures and their transformations. In general, GeoGebra allows algebraic, graphical and tabular presentation of data. It easily provides possibility of manipulating data and comparison of data throughout the different views, construction of geometric figures, representation and analysis of functions, transformations of figures, etc. It is also a powerful tool for justification of mathematical ideas. Based on general features of GeoGebra, the applet enables teachers to justify the formula for calculating the area of a trapezoid easily in a number of ways. At the same time, we used it to explore the ideas and approaches that could be used to prove the formula for the area of a trapezoid, that have been presented in previous section as parts of three strategies. Those strategies should be part of mathematics content knowledge for teaching. Some of the approaches in finding out formula for the area of a trapezoid incorporated in the applet are shown in Figure 13 (enclosure) and Figure14 (transformation).

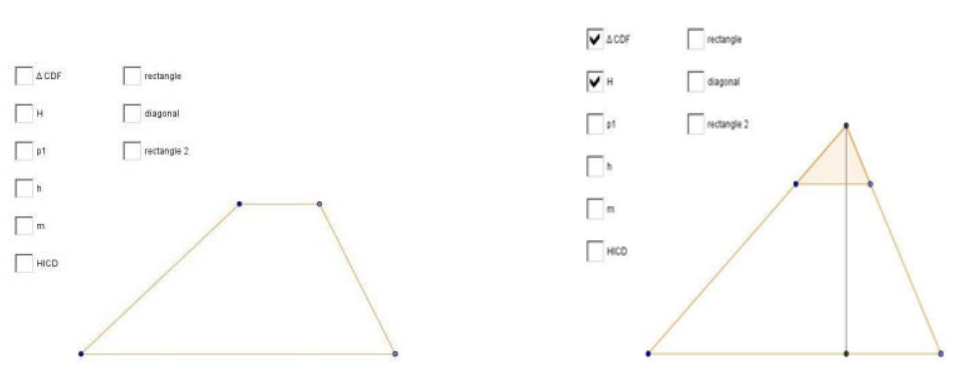

Figure 13. GeoGebra applet (enclosure) 

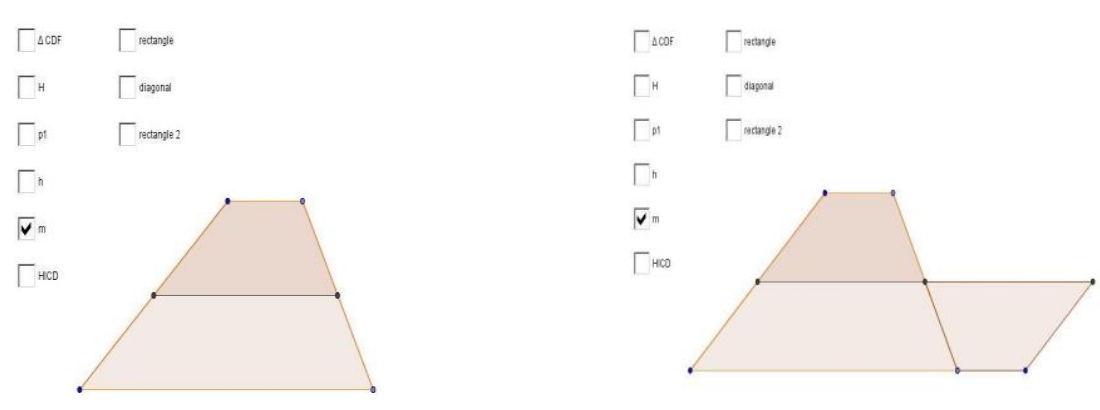

Figure 14. GeoGebra applet (transformation)

\section{Research method}

Solid mathematics content knowledge for teaching enables teachers to, select, design and modify mathematical contents, tasks and problems, establish and revise mathematical goals for students, assess and use resources and tools for teaching, explain mathematical ideas and solve mathematical problems, analyse pupils' mathematical work and thinking. One of the main goals of univeristy mathematics teaching program is to build strong mathematics knowledge of future teachers.

Based on theoretical framework of this paper we have formulated research question: if usage of specially designed GeoGebra applet will influence pre-service mathematics teachers' ways of developing and justifying area of trapezoid.

Nine seniors in bachelor program in mathematics and informatics who were engaged in the course of Practice for teaching mathematics have participated in research. Experiment was conducted in two parts during spring semester 2014 at small university in Bosnia and Herzegovina. At first, students were asked to develop the formula for the area of a trapezoid in as many different ways as much and justify each of them using pen and paper. Answers were clasified in one of three types: type 1 - decomposition, type 2 - enclosure, type $3-$ transformation. In the next step, during 45 minute course session, students had opportunity to explore GeoGebra applet by themselves and find out some of manners of developing the area of trapezoid. Applet offered some possibilities to decompose and transform trapezoid, as well as to enclose trapezoid into triangle. Students are asked to explore given applet and find out ways in development of the formula for the area of a trapezoid. Applet did not include any formula nor did students recieve any explanation from researchers. Their task aftermath was to recognise mathematics ideas and concepts that underlie strategies included in applet and to try to „,invent“ and justify similar ways in developing area of trapezoid. 


\section{Results and discussion}

Students' written answers to the task of developing the formula for the area of a trapezoid in the initial and post-test are analysed. The results of initial and post-test are given in the Table 1 . The post-test part of Table 1 displays only the answers that had not been given in initial test.

Table 1. Students strategies in deriving formula for area of trapezoid

\begin{tabular}{|c|c|c|c|c|c|c|c|c|c|c|c|c|c|c|c|c|c|c|c|c|c|c|c|c|}
\hline \multirow{4}{*}{$\begin{array}{l}\overrightarrow{\vec{E}} \\
\vec{E} \\
\vec{E}\end{array}$} & \multicolumn{12}{|c|}{ Initial test } & \multicolumn{12}{|c|}{ Post test } \\
\hline & \multicolumn{12}{|c|}{ Strategy } & \multicolumn{12}{|c|}{ Strategy } \\
\hline & \multicolumn{4}{|c|}{1.} & \multicolumn{4}{|c|}{2.} & \multicolumn{4}{|c|}{3.} & \multicolumn{4}{|c|}{1.} & \multicolumn{4}{|c|}{2.} & \multicolumn{4}{|c|}{3.} \\
\hline & $\mathrm{a}$ & b & $\mathrm{c}$ & d & $\mathrm{a}$ & $\mathrm{b}$ & $\mathrm{c}$ & d & $\mathrm{a}$ & b & c & d & $\mathrm{a}$ & b & $\mathrm{c}$ & d & $\mathrm{a}$ & $\mathrm{b}$ & $\mathrm{c}$ & $d$ & $\mathrm{a}$ & $\mathrm{b}$ & $\mathrm{c}$ & d \\
\hline 1. & * & + & * & & & + & & & & & & & & & + & & & & A & & & & A & \\
\hline 2. & * & + & * & & & & & + & & & & & & & A & & & A & M & & & M & & \\
\hline 3. & + & + & & & + & & & + & & & & & & & + & & & & + & & & & & $A$ \\
\hline 4. & & & * & & + & + & & & & & & & & + & + & & & & & & + & & & \\
\hline 5. & & & + & & & & & & & & & & & + & & & & & & & & & & \\
\hline 6. & + & + & + & & & & & + & & & & & & + & & & & & & & & + & & + \\
\hline 7. & & & * & & & & & $*$ & & & & * & & + & A & & & & A & & + & 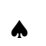 & & + \\
\hline 8. & & + & + & & & & & + & & & & & + & & & & & & A & & & + & + & \\
\hline 9. & & & & & & & & & & & & & 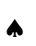 & & & & & & M & & & & & \\
\hline
\end{tabular}

Results of initial test show that one student did not offer any answer to a problem. There are 16 items of strategy decomposition of trapezoid (strategy 1). Each of eight students used strategy of decomposition of trapezoid, five of them decomposing trapezoid into parallelogram and triangle, as in way $1 \mathrm{~b}$ ). Seven students applied decomposing trapezoid by altitudes in rectangle and two right triangles - method 1c). However, four of them applied this method to equilateral trapezoid. The third most used method is strategy 2 approach of enclosing trapezoid into rectangle (2d)): five students came up with this idea and justification, one of them in special case of equilateral trapezoid. Strategy 3 was used only once and in the case of equilateral trapezoid. 
Results of post-test show certan shift in students' reasoning. Students came up with multiple ways of deriving and justifying the formula for the area of a trapezoid. Seven of nine students used strategy 3 in post-test. There were 11 items of deriving the formula using strategy of transformation. Three of them were lacking complete proof, and in one case student presented ideas in irregular manner. Again, students were confident in using strategy 1 . However, method 1d) is not used in any of students' accomplishments.

As the applet did not include all of aforementioned methods in developing the formula for the area of trapezoid, we can claim that there were improvments in students' content knowledge. First of all, in comparision to pre-test answers, all students used more different approaches in finding out and proving the formula after they start to use GeoGebra applet. Some of these approaches haven't been presented in the applet. All but one student used at least one approach from all of three strategies. Several studies have shown that when given an opportunity pupils can come up with multiple ways of discovering or justifying the formula for the area of trapezoid (Manizade, 2012; Wanko, 2005; Young, 2010). Results of our research are consistent to these findings.

\section{Conclusion}

The task of finding a method to derive the formula for the area of any trapezoid involves a wide spectrum of important concepts and ideas in geometry and measurement such as composition and decomposition, transformations, polygons, perpendicular and parallel lines, and similarity and congruency. The task provides the opportunity to explore these ideas and their relationships. Our mini-research gives arguments that engaging pre-service mathematics teachers to develop and justify formula for calculating the area of trapezoid using GeoGebra applets is a worth task that enhances pre-service mathematics teachers' content knowledge for teaching. Teachers' confidence with different approaches which reveal this formula is prerequisite for asking the pupils to do the same task. Mathematics teachers' content knowledge for teaching should be strong enough to let them iniciate pupils' explorations of multiple ways in discovering and justifying formula for area of trapezoid. Therefore, education of future teachers of mathematics should include their involvement in tasks similar to one described in this paper.

\section{References}

Hitt Espinosa, F. (1997). Researching a Problem of Convergence with Mathematica: History and Visualisation of a Mathematical Idea. International Journal of Mathematical Education in Science and Technology, 28(5), 697 - 706.

Manizade, A., \& Mason, M. (2012). A chalenge for all: Developing the area of a trapezoid, Proceedings of 12th International Congress on Mathematical Education (pp. 22892297). Seoul, Korea.

Somayajulu, R. (2012). Investigating pre-service teachers' mathematical knowledge for teaching geometry at secondary level, Proceedings of 12th International Congress on Mathematical Education, Seoul, Korea, 2311 - 2318. 
Wanko, J. J. (2010). Tapping into Trapezoids, Mathematics Teacher, 99(3), 190 - 195.

Young, E. (2010). Trapezoids to Triangles, Mathematics Teaching in the Middle School, $15(7), 414-417$.

Zimmerman, W., \& Cunningham, S. (1991). Editors introduction: What is mathematical visualisation? In W. Zimmennan and S. Cunningham (Eds.) Visualisation in Teaching and Learning Mathematics, MAA Notes and Reports Series 19 (pp. 1-7). The Mathematical Association of America: Washington, DC.

\section{Citation Information}

Pjanić, K., \& Liđan, E. (2015). One usage of geogebra in enhancing pre-service mathematics teachers' content knowledge. Turkish Journal of Computer and Mathematics Education, 6(1), 18-30. 